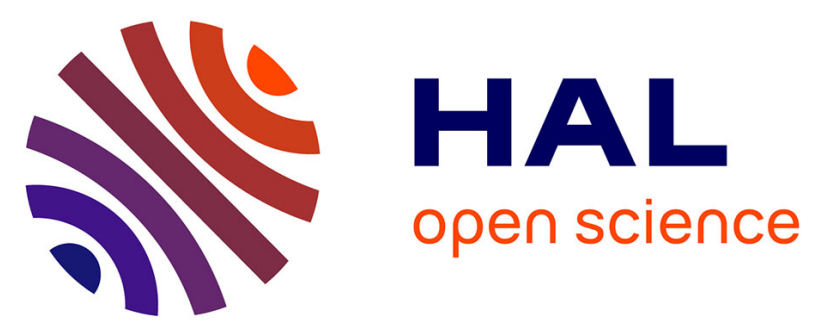

\title{
Study of the Influence of the Source Quality on the Determination of the Shape Factor of Beta Spectra
}

Cindy Le Bret, Martin Loidl, Matias Rodrigues, Xavier Mougeot, Jacques Bouchard

\section{- To cite this version:}

Cindy Le Bret, Martin Loidl, Matias Rodrigues, Xavier Mougeot, Jacques Bouchard. Study of the Influence of the Source Quality on the Determination of the Shape Factor of Beta Spectra. Journal of Low Temperature Physics, 2012, 167 (5-6), pp.985-990. 10.1007/s10909-012-0607-6 . cea-02475351

\section{HAL Id: cea-02475351 https://hal-cea.archives-ouvertes.fr/cea-02475351}

Submitted on 13 Feb 2020

HAL is a multi-disciplinary open access archive for the deposit and dissemination of scientific research documents, whether they are published or not. The documents may come from teaching and research institutions in France or abroad, or from public or private research centers.
L'archive ouverte pluridisciplinaire HAL, est destinée au dépôt et à la diffusion de documents scientifiques de niveau recherche, publiés ou non, émanant des établissements d'enseignement et de recherche français ou étrangers, des laboratoires publics ou privés. 
C. Le-Bret ${ }^{1}$ * M. Loidl $^{1}$ * M. Rodrigues ${ }^{1}$.

X. Mougeot $^{1}$. J. Bouchard ${ }^{1}$

\title{
Study of the influence of the source quality on the determination of the shape factor of beta spectra
}

17.07.2011

\begin{abstract}
We are developing metallic magnetic calorimeters for beta spectrometry with the aim of determining the shape factors of beta spectra. The beta emitter is enclosed inside the detector absorber and the absorber size is chosen such that the detection efficiency is close to $100 \%$ for the entire spectrum.

The nature of the deposit of the beta emitter is critical for precise measurement. A dependence of the shape of the spectrum on the quality of the source has been observed, supposedly due to interactions between the electrons and the deposit itself. We chose for this comparative study a nuclide with an allowed transition whose theoretical spectrum is relatively well-known, ${ }^{63} \mathrm{Ni}$, a pure beta emitter with an endpoint energy of $66.98 \mathrm{keV}$.

Results of measurements of ${ }^{63} \mathrm{Ni}$ deposited with different techniques are presented and compared to each other and to the theoretical spectrum.
\end{abstract}

Keywords Metallic magnetic calorimeters, ionizing radiation metrology, nuclear data, beta spectrometry

PACS numbers: 07.20.Mc, 29.40.Vj, 29.30 Dn,07.81.+a, 23.40.-s, 06.20.-f

\section{Introduction}

In the field of ionizing radiation metrology, the determination of nuclear and atomic data is essential. Therefore, we are developing metallic magnetic calorimeters (MMCs) $)^{1,2}$ for beta spectrometry, with the aim of determining the shape of beta spectra. Shape factors are calculated by comparison between experimental and theoretical spectrum, in order to quantify the discrepancy between theory and experiment. These shape factors have a great importance for beta emitters decaying via forbidden transitions because the theoretical calculation of the corresponding spectrum is complicated ${ }^{3}$ and experimental data are rare and not precise

1:CEA, LIST, Laboratoire National Henry Becquerel, Gif-Sur-Yvette, F-91191, France

E-mail: cindy.le-bret@cea.fr 
enough. In fact, beta spectra are quite difficult to measure precisely with lithium drifted silicon ( $\mathrm{Si}: \mathrm{Li}$ ) detectors or electrostatic or magnetic spectrometers due to low detection efficiency, non-linear response of the detector, or energy loss in the source and/or in the dead layer at the surface of Si:Li detectors.

Metallic magnetic calorimeters with the beta source embedded in the absorber seem promising for improving the determination of shape factors. In addition to the $4 \Pi$ solid angle, the high stopping power of gold will allow for a detection efficiency better than $99 \%$ over the entire spectrum and a threshold at around 1 $\%$ of the endpoint energy. Besides, it has been demonstrated that the linearity of MMCs can be better than $0.5 \%$ over two orders of magnitude in energy ${ }^{4}$.

The first step of the development is to verify the potential of our detection system for the measurement of beta spectra. We chose for this validation ${ }^{63} \mathrm{Ni}$ which has an endpoint energy of $66.98 \mathrm{keV}$ and decays via an allowed transition. The theoretical spectrum is considered well-known and precise experimental spectra have already been published ${ }^{5}$.

Our first experiments show significant differences between the spectra for different beta sources, even for the same technique of source preparation. The first results of the study on the influence of the nature of the source on the shape of the spectrum are presented.

\section{Experimental details}

All the beta sources presented here have been made from a single solution of nickel chloride, with a massic activity of $49 \mathrm{kBq} / \mathrm{g}, 10 \mu \mathrm{g} / \mathrm{g}$ of non-active nickel chloride and $0.1 \mathrm{~mol} / \mathrm{L}$ of hydrochloric acid.

For each of the first two sources, MD6 and MD7, $0.2 \mu \mathrm{L}$ of the solution has been deposited with a micropipette on a gold foil and then freeze dried. We obtained two deposits with different aspects. One of them was invisible whereas the other one exhibited a clearly visible crystallised substance.

In order to see the impact of a thicker deposit, we increased by a factor 5 the amount of non-active $\mathrm{Ni}$ for the same source activity of ${ }^{63} \mathrm{Ni}$. The crystallisation of this deposit, MD8, was also visible. Although the thickness of such an inhomogeneous deposit is difficult to quantify, the mean thickness must be greater by a factor 5, compared to MD6 and MD7.

These three deposits have been made on foils of $10 \mu \mathrm{m}$ thickness. To delimit each Ni deposit, the edges of the gold foils had previously been covered with a silicon glue that was removed after drying of the solution without leaving any trace. Then each deposit was sandwiched with a second gold foil and the two foils were diffusion welded at $670 \mathrm{~K}$.

The last deposit was obtained by electroplating. Since first tests of electroplating on gold were not conclusive, we proceeded to electroplate on copper. In this attempt, the cathode and the anode were both $\mathrm{Cu}$ foils and a voltage of $3.1 \mathrm{~V}(I \sim$ $100 \mu \mathrm{A}$ ) was applied during one hour. A shiny deposit was obtained on one side of the $\mathrm{Cu}$ foil, referred to as MD9. This $\mathrm{Cu}$ foil was folded in two, so as to have the deposit inside, and was embedded in a previously folded Au foil.

However, the deposit on the copper foil was clearly visible, which is not consistent with the concentration of the solution; a thickness below $1 \mathrm{~nm}$ was to be expected. A liquid scintillation measurement permitted to verify that radioactive 
nickel had indeed been deposited. The low count rate, confirmed later by MMC measurement, makes us suspect that the visible deposit is rather copper oxide than metallic nickel.

All the absorbers have an approximate size of $1 \mathrm{~mm}^{2} \times(2 \times 10) \mu \mathrm{m}$.

The thermometer made of Au:Er is fixed to the absorber by ultrasonic wedge bonding, thus assuring a metallic contact and a very efficient heat transfer. The detector (absorber + thermometer) is then placed on the SQUID chip, with the thermometer placed inside the SQUID sensing loop (FIG.1).

The thermal link, which restores the thermal equilibrium, consists in a gold wire (5 $\mu \mathrm{m}$ diameter, $\sim 800 \mu \mathrm{m}$ length) wedge bonded to the absorber and to the copper detector holder.

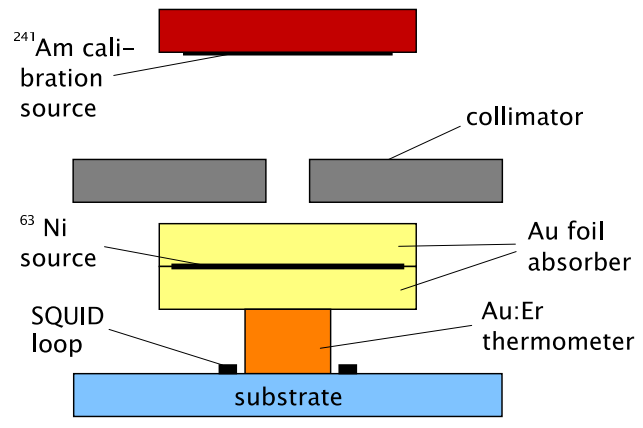

Fig. 1 Schematic of the MMC enclosing a ${ }^{63} \mathrm{Ni}$ source and integrated with an external ${ }^{241} \mathrm{Am}$ calibration source.

The magnetic field required to magnetize the Au:Er sensor is initially being created with a superconducting coil and then maintained by a superconducting lead ring without injection of a current from outside the cryostat.

The detector together with the readout SQUID is operated in a dilution refrigerator at a temperature below $12 \mathrm{mK}$.

For energy calibration, an external ${ }^{241} \mathrm{Am}$ source is used. A thin gold foil $(60 \mu \mathrm{m})$ between the source and the detector stops the $\alpha$-rays and the numerous Np X-rays which would cover a large part of the beta spectrum. Only the $59.5 \mathrm{keV} \gamma$-rays interact in the absorber.

\section{Results}

Data are recorded continuously, at a sampling rate of $100 \mathrm{kHz}$, and the analysis including triggering is done offline via a Matlab code. The pulses of absorbers with freeze-dried sources have a rise time of $\sim 10 \mu$ s and a decay time (1/e) of $3.5 \mathrm{~ms}$, whereas the pulses of MD9 have a rise time of $250 \mu \mathrm{s}$ and a decay time of $13 \mathrm{~ms}$. This difference in time constants may be due to the presence of (ferromagnetic) nickel in the absorber.

A band-pass filter is used to reduce noise and rapidly bring the baseline back to 
zero, i.e minimize pile-up. The energy of each particle interaction is then simply obtained by the determination of the maximum of each pulse. This method implies the pulse shape to be homogeneous, which is confirmed by a systematic pulse shape analysis on additional data recorded at a sampling rate of $2.5 \mathrm{MHz}$. A dead time of $6 \mathrm{~ms}$ and a (software) trigger threshold at $120 \mathrm{eV}$ are implemented. A rejection of pulses with a duration above threshold shorter than $0.16 \mathrm{~ms}$ is added to avoid noise trigger.

In order to validate the spectrum obtained with this analysis, it was compared with two other types of analysis. One of them is based on a matched filter. The other one employs a delay line, which consists in subtracting, with a fixed delay, the opposite of the signal from the original signal to quickly restore the baseline. Then, a Gaussian fit is used to obtain the maximum of the pulse. The spectra obtained with the three analyses are all consistent.

The experimental spectra are compared to a theoretical spectrum, which is calculated by Betashape ${ }^{3}$, a code developed in our institute, which analytically computes the energy spectrum of electrons from beta disintegrations. The beta decay of ${ }^{63} \mathrm{Ni}$ is an allowed transition. Three corrections are taken into account: the influence of the size of the nucleus, the influence of the electron cloud and the radiative effects. In this study of ${ }^{63} \mathrm{Ni}$, the exchange effect is also included in the theoretical spectrum. The exchange effect corresponds to the creation of a beta electron into
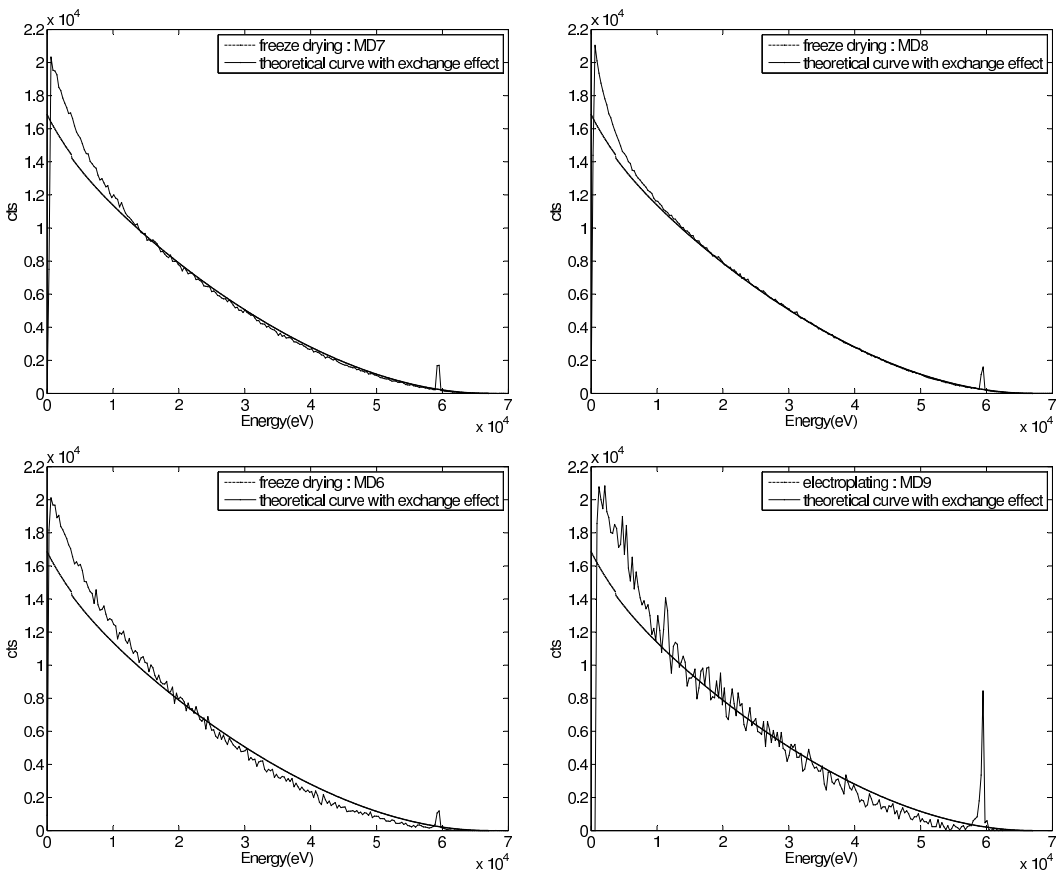

Fig. 2 Measured spectra from different ${ }^{63} \mathrm{Ni}$ sources compared to a theoretical spectrum. Top: freeze-dried deposits with visible crystallisation (MD7 and MD8), bottom: freeze-dried thin deposit (MD6) and electroplated deposit (MD9). All the spectra have been normalized with respect to MD8. 
a bound orbital of the daughter atom, with the simultaneous ejection of a corresponding shell electron. This effect leads to a deviation of the spectrum at low energy. The enhancement had been calculated from 5 to $67 \mathrm{keV}^{6}$. These values were fitted and the fit function was extrapolated down to $0 \mathrm{keV}$. This fit is applied to correct the theoretical spectrum. A calculation of the exchange effect for lowest energies is about to be implemented in Betashape, which will allow us to validate this fit.

The measured spectra indicate that the thickness of the deposit has a great influence on the spectrum shape (FIG.2). MD7 and MD8, whose spectra are considered to be consistent with each other, showed a visible crystallisation which may partially explain the deviation at low energies by the creation of athermal states in the crystallised source and hence a shift towards lower energies. The spectra of MD9 and MD6, which are not consistent with whose of MD7 and MD8 are in turn rather compatible with each other. In a purely metallic electroplated source, the entire beta energy should be converted to heat, but the supposed copper oxide of MD9 may play the same role as a thin salt deposit as it should be present in MD6.

Unlike what one could expect, a thick deposit seems to yield a spectrum more consistent with the theory than a thin one. We have already observed this phenomenon in a previous measurement of ${ }^{241} \mathrm{Pu}^{8}$. A ${ }^{241} \mathrm{Pu}$ deposit thicker than the ${ }^{63} \mathrm{Ni}$ sources gave a spectrum in good agreement with the theoretical spectrum within two third of the energy range but showed a discrepancy at low energy.

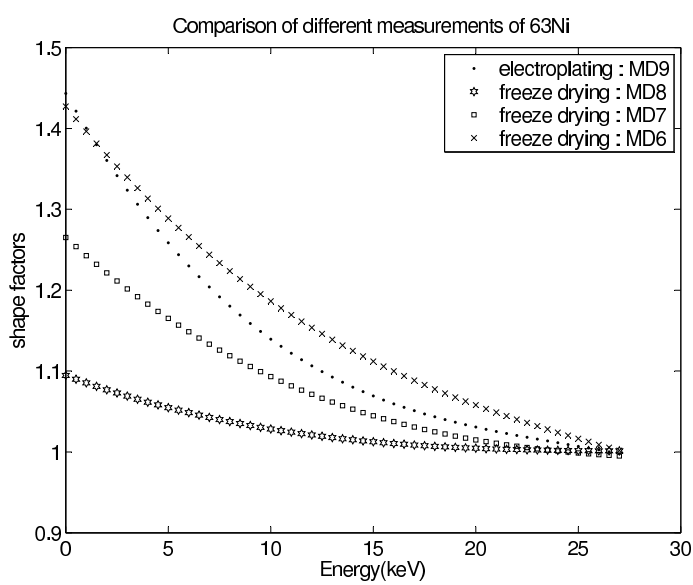

Fig. 3 Comparison of shape factors for different ${ }^{63} \mathrm{Ni}$ measurements. Only the low energy part is presented.

The differences between the measured spectra are even more clearly visible in the corresponding shape factors (FIG.3). In principle, a shape factor should be calculated from the comparison between theory and a correct experimental spectrum. That is to say that for one beta emitter, only one shape factor should be established.

The differences between our experimental spectra make it impossible to obtain a 
single shape factor. This makes it evident that a type of source without any influence on the spectrum shape is yet to be found.

\section{Conclusions and perspectives}

The measurements of beta spectra of different ${ }^{63} \mathrm{Ni}$ sources have clearly demonstrated an influence of the nature of the source deposit on the spectrum shape and hence on the determination of the shape factor. To perform a precise measurement of beta spectra with metallic magnetic calorimeters, one has to assure that the measured temperature rise is strictly proportional to energy. In this regard, the thickness and the nature of the source are essential.

This observation leads us to plan two extreme measurements: with a deposit thick enough to stop beta particles of the entire energy range, and a deposit as thin as possible to be able to consider that none of the electrons interact in the source itself. Concerning a thick deposit, a mixture of the solution with a resin or epoxy is planned. To obtain a thinner deposit, two methods are envisaged : on the one hand, electroplating on gold. An appropriate increase of Ni concentration and current might avoid the difficulties of the first attempts. On the other hand, we intend to implant the beta emitter into the absorber matrix. Implantation is probably the most promising source preparation technique, but more time consuming to realize due to the necessity of having access to a mass separator.

\section{References}

1. C. Enss et al., J. Low Temp. Phys., 121, (2000) 137-176.

2. A. Fleischmann, C. Enss and G. Seidel, "Metallic Magnetic Calorimeters", in Cryogenic Particle Detectors, Topics Appl. Phys. 99, C. Enss (Ed.), Springer Berlin/Heidelberg (2005) 151-216.

3. X. Mougeot et al., "Calculation of beta spectra for allowed and unique forbidden transitions", submitted to Proc. of Advances in Liquid Scintillation Counting (LSC 2010), Paris, France, 610 Sept. 2010, to be published in Radiocarbon (2011).

4. M. Rodrigues et al., J. Low Temp. Phys. 151 (2008) 1080.

5. L.C. Angrave et al., Proc. of the 7th International Workshop on Low Temperature Detectors (LTD-7), Munich, Germany, 27 July - 2 Aug. 1997, Ed. S. Cooper, Munich (1997) 178.

6. L. Angrave, $\mathrm{PhD}$ Thesis on "The physics of non-equilibrium phonons and non-equilibrium superconductivity applied to a precision measurement of the beta spectrum of ${ }^{63} \mathrm{Ni}$ ", Trinity Term 2000, Madgalen College, Oxford (2000).

7. A. Burck et al., J. Low Temp. Phys., 151 (2008) 337-344.

8. M. Loidl et al., Proc. of the 13th International Workshop on Low Temperature Detectors, LTD-13, 20-24 July 2009, Stanford, USA, AIP Conference Proceedings 1185 (2009) 587. 